\title{
\& \\ Vacancy-induced magnetism in graphene and graphene ribbons
}

\author{
J. J. Palacios and J. Fernández-Rossier \\ Departamento de Física Aplicada, Universidad de Alicante, San Vicente del Raspeig, E-03690 Alicante, Spain \\ L. Brey \\ Instituto de Ciencia de Materiales de Madrid, Consejo Superior de Investigaciones Científicas, E-28049 Cantoblanco, Spain
}

(Received 14 February 2008; revised manuscript received 27 March 2008; published 19 May 2008)

\begin{abstract}
We address the electronic structure and magnetic properties of vacancies and voids both in graphene and graphene ribbons. By using a mean-field Hubbard model, we study the appearance of magnetic textures associated with removing a single atom (vacancy) and multiple adjacent atoms (voids) as well as the magnetic interactions between them. A simple set of rules, based on the Lieb theorem, link the atomic structure and the spatial arrangement of the defects to the emerging magnetic order. The total spin $S$ of a given defect depends on its sublattice imbalance, but some defects with $S=0$ can still have local magnetic moments. The sublattice imbalance also determines whether the defects interact ferromagnetically or antiferromagnetically with one another and the range of these magnetic interactions is studied in some simple cases. We find that in semiconducting armchair ribbons and two-dimensional graphene without global sublattice imbalance, there is a maximum defect density above which local magnetization disappears. Interestingly, the electronic properties of semiconducting graphene ribbons with uncoupled local moments are very similar to those of diluted magnetic semiconductors, presenting giant Zeeman splitting.
\end{abstract}

DOI: 10.1103/PhysRevB.77.195428

PACS number(s): 73.22.-f, 75.75.+a

\section{INTRODUCTION}

Magnetic order occurs, in most instances, in materials with partially filled $d$ or $f$ shells. There is, however, a recent awareness that the possibility of magnetic order can also occur in materials without open $d$ or $f$ shells. ${ }^{1-5}$ Experimental evidence of this type of magnetism was found in thin films of certain oxides $\left(\mathrm{HfO}_{2}, \mathrm{ZnO}\right.$, and $\left.\mathrm{TiO}_{2}\right),{ }^{1}$ as well as irradiated graphite $^{2}$ and thiol-capped gold nanoparticles. .,7 $^{6}$

Although more experimental work is probably necessary to confirm and understand magnetism in these systems, there are at least two scenarios for which theory provides a mechanism for the appearance of magnetism without $d$ or $f$ open shells. On one hand, in some lattices, intrinsic lattice defects such as vacancies lead to the formation of local magnetic moments, a preliminary condition for the existence of magnetic order. This is the case in graphite, ${ }^{8}$ graphene, ${ }^{9-11}$ and II-VI semiconductors. ${ }^{12}$ On the other hand, it has been recently found that clusters with specific shapes, such as triangular graphene islands ${ }^{3,13}$ or icosahedral ${ }^{4,5}$ gold clusters, have large degeneracies at the Fermi energy in their singleparticle spectra. These degeneracies are related to the symmetry of the nanostructure and, in words of Luo et al., ${ }^{5}$ they behave like "superatoms," with magnetic ground states that comply with atomiclike Hund's rules.

Importantly, both vacancy-induced ${ }^{10,11}$ and superatomic magnetism ${ }^{3}$ occur in graphene structures and, as we show here, have the same origin. In this work, we present extensive numerical work to understand vacancy-induced magnetism in graphene and graphene ribbons and we analyze our results in the context of a broader theoretical framework that unifies superatomic ${ }^{3}$ and vacancy-induced magnetism ${ }^{10,11}$ in graphene. In part, our motivation stems from the recently shown possibility of fabricating high mobility graphenebased field effect transistors, ${ }^{14-19}$ which has created enormous interest in graphene-based electronics. ${ }^{20}$ Additional possibilities arise from the fabrication of semiconducting graphene ribbons ${ }^{21-23}$ and graphene nanoislands ${ }^{20,24}$ with top-down techniques as well as the growth of graphene islands with bottom-up techniques. ${ }^{25,26}$ Magnetic order in patterned or nanostructured graphene would bring up new opportunities of research in spintronics.

Graphene honeycomb structure is a bipartite lattice, which is formed by two interpenetrating triangular sublattices, $A$ and $B$, such that the first neighbors of an atom $A$ belong to the sublattice $B$ and vice versa. ${ }^{27}$ The low-energy electronic structure of graphene can be described by a single-orbital $\left(p_{z}\right)$ nearest-neighbor hopping Hamiltonian. ${ }^{27,28}$ This model correctly describes two dimensional graphene as a zero-gap semiconductor with linear bands around the Fermi energy. The single-particle spectrum of a nearest-neighbor tightbinding model in a bipartite lattice has a particle-hole symmetry. ${ }^{29,30}$

The magnetic properties of both graphene-based nanostructures and defective graphene are intimately related to the appearance of midgap states and how they are affected by electron-electron interactions. The existence of zero-energy states in disordered bipartite lattices was proved by Inui et $a l .{ }^{29}$ Within the first-neighbor tight-binding model, a suffi-

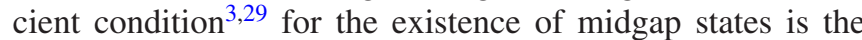
existence of a finite sublattice imbalance, $N_{I} \equiv N_{A}-N_{B}$, where $N_{A}$ and $N_{B}$ are the number of atoms belonging to each sublattice or missing from each sublattice in an otherwise perfect system. Thus, whereas ideal graphene has $N_{I}=0$ and no midgap states, both defective graphene and some graphene islands, such as triangles, can present finite sublattice imbalance and $\left|N_{I}\right|$ midgap states. The result of Inui et $a l .{ }^{29}$ was used in a recent work on disorder in graphene by Pereira et al. ${ }^{31}$ Incidentally, the existence of zero-energy or midgap states in uncompensated graphene structures was known long ago in the context of chemical studies of hydrocarbons as the Longuet-Higgis conjecture. ${ }^{32}$ 
Because of the particle-hole symmetry, midgap states are half-filled for neutral graphene and the appearance of magnetic moments is expected in analogy with Hund's rule in atomic magnetism. The Hubbard model extends the singleparticle tight-binding model including the effect of Coulomb repulsion between two electrons in the same atomic site. Importantly, a theorem by Lieb, ${ }^{33}$ valid for the exact ground state of the Hubbard model and neutral bipartite lattices, states that the total spin $S$ of the ground state is given by $2 S=\left|N_{A}-N_{B}\right|=\left|N_{I}\right|$. Lieb's ${ }^{33}$ theorem provides a rigorous connection between vacancies in the graphene lattice and the emergence of magnetism. As a result, sublattice unbalanced neutral graphene will always present a finite total magnetic moment.

Although Lieb's ${ }^{33}$ theorem provides the total spin of the ground state, it does not say much about the actual local magnetic order or spin texture. For instance, $S=0$ does not preclude the existence of local magnetic moments antiferromagnetically coupled or presenting compensated ferrimagnetic order. The most notorious example of compensated ferrimagnetic order can be found in zigzag ribbons, ${ }^{34-38}$ where each edge presents ferromagnetic order antiparallel to each other for a total vanishing magnetic moment. Other examples can be found in hexagonal graphene islands, where, beyond a critical size, contiguous sides alternate the direction of the ferromagnetically ordered magnetic moments. ${ }^{3}$

The rest of this paper is organized as follows. In Sec. II, we review the single-orbital Hubbard model and the different methodologies used to describe the electronic structure of defective graphene and graphene ribbons. The underlying noninteracting spectrum and associated magnetic textures can be anticipated following some basic rules which are presented in Sec. III. We illustrate the validity of the rules by numerical calculations in the case of semiconducting armchair ribbons (Sec. IV) with vacancies, voids, or notches, both in the noninteracting (Sec. IV) and interacting (Sec. V) cases. The results for bulk graphene are discussed in Sec. VI. Summary and conclusions are presented in Sec. VII.

\section{METHODOLOGY}

We consider the low-energy physics that takes place in the subspace expanded only by the single $p_{z}$ orbital (the one perpendicular to the graphene plane). Next-to-near neighbor hopping is neglected and the electron-electron interactions are locally included in the form of an on-site repulsion or Hubbard model. When the interactions are turned off, this reduces to the widespread one-orbital tight-binding model. ${ }^{28,39-41}$ The Hubbard term is treated in a mean-field approximation. ${ }^{3,34}$ Comparison between the results so obtained and density functional theory (DFT) calculations yield very good agreement for two-dimensional graphene, ${ }^{42}$ carbon nanotubes, ${ }^{42,43}$ zigzag ${ }^{36,38}$ and armchair graphene ribbons, ${ }^{35}$ as well as graphene islands. ${ }^{3}$

We model vacancies and voids in perfect graphene or graphene ribbons by removing atoms, actually, by removing the representing $p_{z}$ orbitals in the tight-binding model. This results in a reduction of the coordination of the atoms adjacent to the missing atoms. We ignore the lattice distortion and we assume that the on-site energy is the same for edge and bulk atoms. The single-orbital Hamiltonian implicitly assumes full hydrogen passivation of the $s p_{2}$ dangling bonds of the atoms without full coordination. This assumption, which might not be completely realistic in the case of actual vacancies, ${ }^{8}$ does not invalidate our model, for we can consider an alternative physical realization: the chemisorption of a hydrogen atom on top of a bulk graphene atom ${ }^{11}$ effectively removes a $p_{z}$ orbital from the low-energy Hamiltonian. In our one-orbital model, there is no difference between these two scenarios.

DFT calculations on graphene ribbons, ${ }^{35,37}$ graphene islands, ${ }^{3,44,45}$ and bulk graphene with vacancies ${ }^{11}$ have shown that the results follow the predictions of Lieb's theorem, even though DFT calculations go beyond the first-neighbor hopping, and short-range interaction Hubbard model on which the theorem is based. In other words, second neighbor hopping and intersite Coulomb repulsion, present in the DFT calculations, do not modify the relation between lattice imbalance and total spin of the ground state warranted for the Hubbard model for which these couplings are absent. From this point of view, these couplings are irrelevant. It is thus justified to consider the following mean-field Hamiltonian:

$$
H=H_{0}+U \sum_{i}\left(n_{i \uparrow}\left\langle n_{i \downarrow}\right\rangle+n_{i \downarrow}\left\langle n_{i \uparrow}\right\rangle\right)-U \sum_{i}\left\langle n_{i \downarrow}\right\rangle\left\langle n_{i \uparrow}\right\rangle,
$$

where $i$ runs over all lattice sites and the noninteracting Hamiltonian reads

$$
H_{0}=\sum_{i, j} t\left(c_{i}^{\dagger} c_{j}+c_{j}^{\dagger} c_{i}\right),
$$

where the sum runs over nearest-neighbor lattice sites $i, j$ and $t=2.5 \mathrm{eV}$. Without loss of generality, we have set the diagonal terms of the Hamiltonian to zero. For neutral grapheme, we can rewrite the mean-field Hamiltonian (up to a constant) as the sum of two terms,

$$
H=H_{0}+\frac{U}{2} \sum_{i} n_{i}\left\langle n_{i}\right\rangle-U \sum_{i} 2 m_{i}\left\langle m_{i}\right\rangle,
$$

where $m_{i}=\frac{1}{2}\left(n_{i \uparrow}-n_{i \downarrow}\right)$ and $n_{i}=n_{i \uparrow}+n_{i \downarrow}$. The second term in $H$ represents the nontrivial contribution of interactions.

The calculations have been performed considering three different types of boundary conditions. For the evaluation of the noninteracting density of states (DOS) in ribbons, we compute the Green's function projected on the region where the defects are located. The perfect regions of the ribbon away from the defects are included in the Green's function by means of a self-energy. We refer the reader to Ref. 41 for more details on this methodology. When the interactions are turned on $(U \neq 0)$, we consider ribbons with periodic boundary conditions in one direction or, for bulk graphene, periodic boundary conditions in both directions. More details will be given in the respective sections.

\section{BASIC RULES}

In this section, we provide some general rules to understand the appearance of midgap states and magnetic textures 


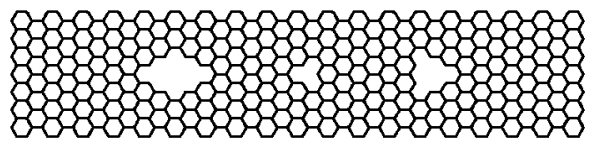

FIG. 1. Examples of voids with different sublattice imbalance charges in the middle of a graphene ribbon. From left to right, the associated imbalance charges are $N_{I}=0,-1$ (vacancy), and 2 .

due to single-atom vacancies or, more generally, voids in an otherwise sublattice balanced graphene structure, for instance, an infinite defect-free semiconducting armchair ribbon. A void in graphene can be partially characterized by the number and type, $A$ or $B$, of atoms removed from the otherwise perfect structure. We will label voids as one would do for chemical compounds, $A_{N_{A}} B_{N_{B}}$. Voids will be unbalanced when they are created by removing $N_{A}$ and $N_{B}$ atoms such that $N_{I}=N_{A}-N_{B} \neq 0$. The sublattice imbalance $N_{I}$, which can be either positive or negative, can be interpreted as an imbalance "charge." This quantity is central to our discussion, although the exact formula of the void is also important since it gives an idea of the size and shape of the void. In the case of ribbons, the voids can be close to the edges, thus becoming notches. (For the most part, we will refer to voids in the bulk of the ribbon, but the conclusions equally apply to the case of notches on the edges.) For a single unbalanced void characterized by $N_{I},\left|N_{I}\right|$, zero-energy states appear in the noninteracting spectrum with weight on only one sublattice. ${ }^{29}$ When the graphene structure presents a gap $E_{g}$, as in armchair ribbons, these states are normalizable and localized around the void (in contrast to two-dimensional graphene where the zero-energy states are not normalizable ${ }^{46}$ ). Figure 1 shows various examples of voids in a ribbon with a different sublattice imbalance $N_{I}$.

Now, let us consider two voids locally characterized by $N_{I}(1)$ and $N_{I}(2)$, which are sufficiently separated so that they do not affect each other. According to the result of Inui et al. ${ }^{29}$ the single-particle spectrum has, at least,

$$
N_{Z}^{\min }=\left|N_{I}(1)+N_{I}(2)\right|
$$

midgap states. The important question is what happens when the distance between them is such that they do affect each other. If $N_{I}(1)$ and $N_{I}(2)$ have the same sign, there are $\left|N_{I}(1)\right|+\left|N_{I}(2)\right|$ midgap states regardless of the distance. If they have different signs, e.g., $N_{I}(1)+N_{I}(2)=0$, Eq. (4) apparently warrants the annihilation of midgap states. Within the noninteracting model, midgap states are $100 \%$ sublattice polarized. The noninteracting Hamiltonian has finite matrix elements between states that have weight on different sublattices. Hence, the mechanism for midgap state annihilation is the hybridization of midgap states localized in different sublattices. This annihilation occurs as bonding-antibonding pairs of midgap states form, resulting in a shift in their energy and in a loss of the sublattice polarization. For large distances, however, this annihilation does not occur.

A well understood related example occurs in zigzag ribbons. ${ }^{39,40,47}$ The edge of a zigzag ribbon has a local sublattice imbalance. If the top edge belongs to the $A$ sublattice, the bottom edge belongs to the $B$ sublattice. States fully lo- calized in the edge have zero energy and are localized in a single sublattice. To the extent that states mostly localized on the top edge penetrate into the ribbon, they hybridize with states mostly localized on the bottom edge. This mixing results in a bonding-antibonding splitting that takes these states away from the Dirac energy. In the case of zigzag ribbons, the degree of localization in the unit cell depends on the wave vector. States close to the zone boundary are very localized in the edges and have energy very close to zero. ${ }^{39,40,47}$ The localization decreases as the wave vector departs from the boundary, resulting in the hybridization and the departure from zero energy. ${ }^{39}$

In the general case, one can conclude that the minimum number of zero-energy states will be given by

$$
N_{Z}^{\min }=\sum_{\alpha, \beta}\left|N_{I}(\alpha)+N_{I}(\beta)\right|
$$

where the integer indices $\alpha$ and $\beta$ run over voids with the same imbalance sign, respectively. In practice, within an arbitrarily small energy interval $|E| \rightarrow 0$, the number of zeroenergy states can be as large as

$$
N_{Z}^{\max }=\sum_{\alpha}\left|N_{I}(\alpha)\right|+\sum_{\beta}\left|N_{I}(\beta)\right|
$$

In the general case, $N_{Z}$ will be a number between $N_{Z}^{\min }$ and $N_{Z}^{\max }$.

When electron-electron interactions are turned on, at least locally in the form of a Hubbard-type interaction, Lieb's ${ }^{33}$ theorem guarantees that $\left|N_{I}\right|=2 S$ for neutral graphene. The theorem, however, does not exclude the possibility of spinsymmetry broken local magnetic order even when $S=0$ or smaller. For instance, two or more voids with local sublattice imbalances that cancel out the total imbalance can still retain their local magnetic order when they are not in proximity. When the imbalance of the void is zero but the size is large, an internal ferrimagnetic order cannot be discarded either. In general, calculations will be necessary to ascertain the spin texture in these situations. A few conclusions, however, can be reached without actually performing any calculations. One can distinguish four cases.

(1) $N_{Z}^{\min }=N_{Z}=N_{Z}^{\max }$. In this case, all the voids are of the same sign. The coupling between them is always ferromagnetic and the spin of the ground state is $2 S=N_{Z}$. The splitting with smaller spin states will depend on the intervoid coupling.

(2) $N_{Z}^{\min }=N_{Z}<N_{Z}^{\max }$. In this case, all the voids of different signs are in proximity and interact, yielding a $2 S=N_{Z}^{\mathrm{min}}$ state. Calculations will be necessary to ascertain the spin texture in these situations.

(3) $N_{Z}^{\min }<N_{Z}=N_{Z}^{\max }$. In this case, all the voids of different types are separated and uncoupled. The ground state has $2 S=N_{Z}^{\min }$, but the spin-flip gap is negligible since there are uncoupled magnetic moments.

(4) $N_{Z}^{\min }<N_{Z}<N_{Z}^{\max }$. In this case, there are voids of different signs, but some of them are uncoupled and some are not. This is the most general case. The ground state has $2 S$ $=N_{Z}^{\min }$, but, as in the previous case, the spin-flip gap is neg- 

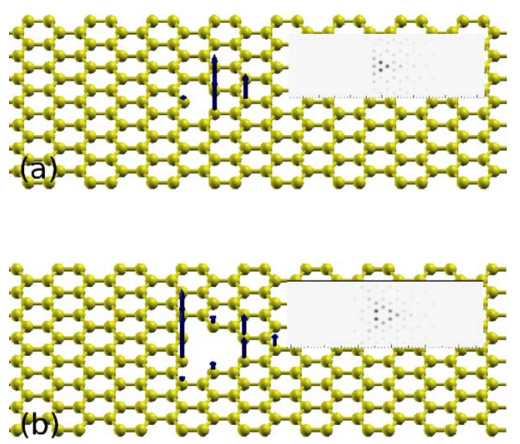

FIG. 2. (Color online) (a) Magnetic moments on lattice sites around a single vacancy. Inset: Probability density of the zeroenergy state built with Gaussian functions on lattice sites. (b) Same as in (a) but for a triangular void with $N_{I}=2$. Inset: Same as in (b) but summing over the two zero-energy states.

ligible since there are uncoupled magnetic moments. Calculations will be necessary to ascertain the spin texture in these situations as well.

\section{DEFECTS IN SEMICONDUCTING GRAPHENE RIBBONS: NONINTERACTING THEORY}

In this section, we study the electronic structure of defective graphene armchair ribbons within the noninteracting tight-binding model. The results are obtained by using the cluster embedded method described in Ref. 41. In this methodology, a finite portion of the ribbon containing the defects is attached to two semi-infinite perfect ribbons of the same width and compute the DOS of the defective region. In the defect-free case, we obtain a gap in the DOS. We consider armchair graphene ribbons of width $W=N_{y} a$, where $N_{y}$ is an integer number and $a=2.42 \AA$ is the graphene lattice parameter. We only consider values of $W$ such that, within the first-neighbor tight-binding model, the ribbon is semiconducting. ${ }^{39,40}$ This happens if $N_{y}+1$ is not a multiple of 3. More realistic calculations ${ }^{35,48}$ predict that, because of lattice distortion of the edge atoms, even ribbons with $N_{y}$ $+1=3 m$, where $m$ is an integer, are semiconductors. Semiconducting graphene ribbons attract interest due to possible applications in nanoelectronics. ${ }^{21,22,49,50} \mathrm{As}$ in the case of $\mathrm{Si}$ based semiconductors, their electronic structure might be strongly influenced by impurities. Here, we study the effect of vacancies and voids, which are expected to act as neutral impurities.

\section{A. Single void}

The simplest defective structure is a perfect semiconducting graphene ribbon from which a single atom, $A$ or $B$, is removed. In agreement with Eq. (4), a zero-energy state appears in the DOS. For neutral graphene, this state is halffilled. In other words, a spin unpaired electron occupies the midgap state. The local density of states at zero energy, which is nothing but the modulus square of the wave function associated with the zero-energy state, is shown in the inset of Fig. 2(a). The state is localized in the neighborhood of the vacancy. The shape of the midgap state is also pecu- liar: it has a clear directionality. Monatomic vacancies have the shape of a triangle. Two vertices of the triangle point toward the edges of the ribbon, whereas the lateral vertex can point downstream or upstream along the ribbon. The midgap state is peaked around the lateral vertex. Hence, midgap states have a strong directional character.

Importantly, the integrated charge, including both midgap and band states below the Fermi energy, yields a homogeneous charge distribution: there is one electron per atom in every atom even in the presence of the vacancy. Hence, the localized midgap state does not imply charge localization; yet, there is a finite spin density. The total spin $S$ of the neutral graphene with one vacancy is $1 / 2$ and the spin density does show a nonhomogeneous texture, as shown in Fig. 2(a). Hence, the region of the material around the defect has no local charge but has local spin.

Our next step is to consider larger voids. In Fig. 2(b), we show the results for a void with $N_{I}=2$ obtained by removing four atoms $\left(A_{3} B_{1}\right)$. In agreement to Eq. (4), there are two midgap states (per spin). Their local density of states is shown in the inset of Fig. 2(b). As in the case of monatomic vacancy, two electrons occupy the midgap states. The integrated local charge is also homogeneous: one electron per site. Within the framework of the noninteracting model, we cannot discriminate between $S=0$ or $S=1$. As we will discuss below, when Hubbard repulsion is turned on, Lieb's ${ }^{33}$ theorem warrants that the spin of this structure is $S=1$. In Fig. 2(b), we show the magnetization density calculated including the interactions, as will be discussed below. As in the case of a single missing atom, there is a magnetic texture with $S$ $=1$, which is localized in a region without localization of extra charge. Triangular voids maximize $\left|N_{I}\right|$ while removing the minimum number of atoms. Larger ones $\left(\left|N_{I}\right|>2\right)$ exhibit similar features to the ones already discussed. More complicated voids with zigzag edges such as hexagons or rhombi, which have $N_{I}=0$, can still present quasi-zero-energy states if they are sufficiently big and therefore might exhibit spin textures as discussed below.

\section{B. Two voids}

As a step toward understanding the electronic structure of graphene with a finite density of defects, we first consider the electronic structure of two voids with the same absolute value of $N_{I}$. Each void has a well defined sublattice imbalance number $N_{I}$ when apart, which can be positive or negative. If the sublattice imbalance of the two voids has the same sign, the global structure has twice as many zeroenergy states as the separated defects. The noninteracting Hamiltonian does not couple sites on the same sublattices so that the zero-energy states associated with two vacancies with sublattice imbalance of the same sign cannot interact regardless of the distance separating them, i.e., the noninteracting DOS will always present a delta function at zero energy that can accommodate $2 \times\left|N_{I}\right|$ electrons per spin channel.

When the imbalance numbers are of different signs, they cancel out each other. When the defects are far away from each other, their local electronic structure is expected to be 


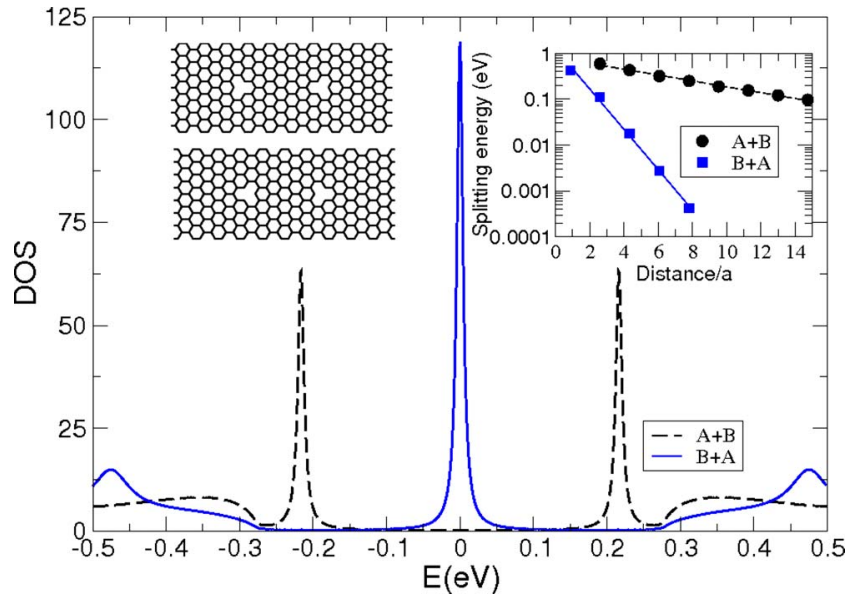

FIG. 3. (Color online) Density of states near the Dirac point for an armchair ribbon of $W=7 a$ with two vacancies presenting imbalance charge of different signs and same modulus $\left(N_{I}= \pm 1\right)$. The solid lines correspond to the $B+A$ case (left lower inset) and the dashed lines correspond to the $A+B$ case (left upper inset). $A$ finite broadening has been added for visibility's sake of the delta functions. The finite, but small energy splitting in the former case is not visible for this broadening. Right inset: Bonding-antibonding energy splitting as a function of the distance between vacancies for the two different spatial orderings.

the same as that of a single defect: midgap states localized in a single sublattice around the missing atoms. As the defects become closer, the single-particle Hamiltonian, which couples atoms of different sublattices, will hybridize the otherwise sublattice polarized midgap states, which will result in bonding and antibonding pairs away from zero energy. The localization length of the single-defect states sets the length scale at which this hybridization occurs.

Our numerical calculations confirm this scenario. For a given width, the hybridization depends on the distance and, given the directional character of the midgap states in ribbons, on the relative orientation.

In Fig. 3, we show the DOS for a system with two monatomic vacancies $A$ and $B\left(N_{I}= \pm 1\right.$, respectively). They are aligned along the ribbon axis and placed at a distance of $6.35 a$ away from each other for the two possible spatial orderings, $A+B$ (head to head) and $B+A$ (tail to tail), as shown in the left insets. Due to the high directional character of the associated zero-energy states, the coupling is not invariant against the interchange of positions and the zero-energy states differently hybridize, depending on the spatial ordering. In one case, the twofold zero-energy peak clearly splits into two above and below the Fermi energy. In the other, the splitting is much smaller (not visible in this scale). For one relative orientation, the wave functions overlap and the degeneracy is strongly removed. For the other, the wave functions do not couple at this distance and the degeneracy is practically unaffected. In the right inset of Fig. 3, we show a logarithmic plot of the energy splitting as a function of the distance for the two cases. The splitting exponentially decays in both, reflecting the localized character of the zero-energy states.

We now consider the case of pairs of defects with larger sublattice imbalance. Figure 4 shows the DOS for two trian-

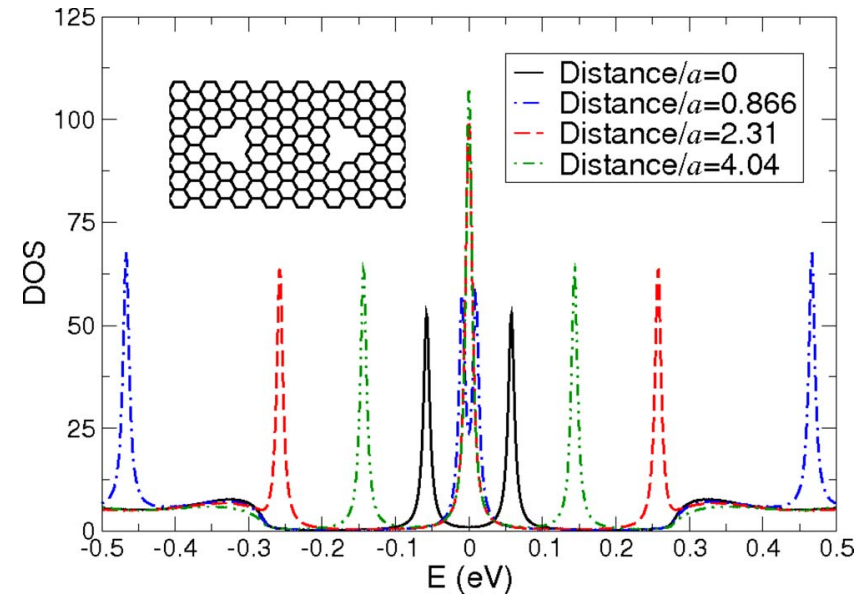

FIG. 4. (Color online) DOS projected on the vicinity of two triangular voids placed along the axis of a semiconducting ribbon for different relative distances. The imbalance charges are the same but differ in sign $\left(N_{I}= \pm 2\right)$.

gular voids characterized by $N_{I}=+2$ and $N_{I}=-2\left(A_{3} B_{1}\right.$ and $A_{1} B_{3}$, respectively) at different distances. We have selected only one possible ordering in this case (tail to tail). According to these sublattice imbalances, each void has associated two localized states. These two states also present a strong directional character but is different for the two. This can be inferred from the two different bonding-antibonding splitting energies for a given distance seen in Fig. 4. We note that even when the voids approach each other, the splitting associated with one of the localized states remains small, still being practically zero for small distances. Only in the extreme limit of zero distance when the two voids merge into a single one with sublattice imbalance $N_{I}=0\left(A_{4} B_{4}\right)$, there are no zero-energy states.

As the sublattice imbalance of the merging voids becomes bigger and these condense into even bigger $N_{I}=0$ voids, the number of states that appear in a vicinity of zero $|E| \rightarrow 0$ increases with the charge of these. Since the appearance of magnetic order relies on the existence of zero-energy states, large voids with $N_{I}=0$ can still present ferrimagnetic order, the only condition being that they are formed out of voids with large sublattice imbalance. In other words, their contours must present sufficiently long zigzag sections. This limits the possible shapes of these voids to, e.g., rhombohedral (see Fig. 9) or hexagonal forms. This conclusion is no different from that reached on graphene hexagonal islands ${ }^{3}$ or finite length ribbons, ${ }^{44}$ where calculations have revealed compensated ferrimagnetic order developing along the edge beyond a certain critical size.

Finally, in order to stress the fact that there is nothing in the previous discussion specific to voids in the bulk of the ribbon, we compute the noninteracting DOS for an $A_{6} B_{4}$ void plus an $A B_{2}$ void placed on the edges (i.e., notches) with $N_{I}=2$ and $N_{I}=-1$, respectively (see Fig. 5). Removing just one atom to create a notch with $N_{I}=-1$ would have given the same charge as the $A B_{2}$ defect, but it would be chemically very unstable and we ignore that possibility. The notches are located on opposite edges, although the results apply the same for notches on the same edge. A single doubly degen- 


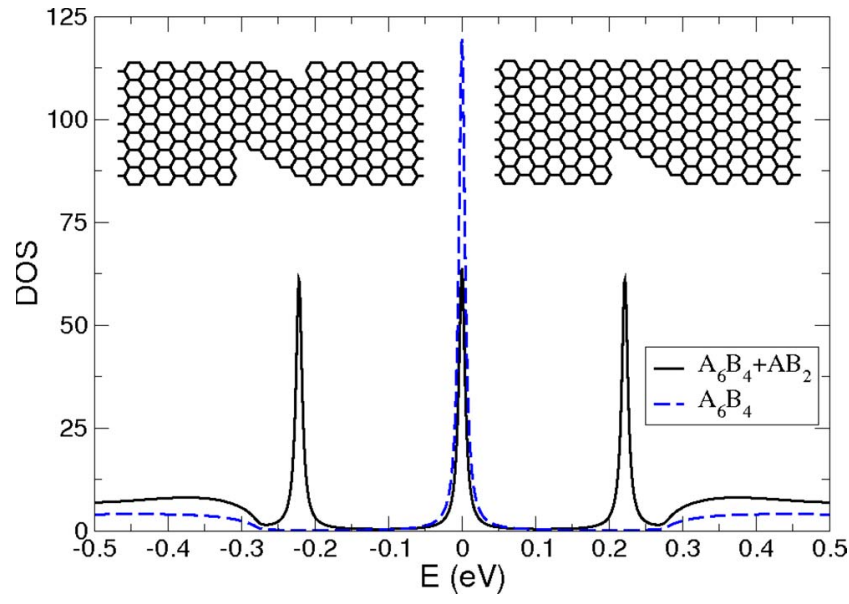

FIG. 5. (Color online) DOS for a single notch with imbalance charge $N_{I}=2$ (blue dashed line, right inset). The same notch with an additional notch nearby of charge $N_{I}=-1$ (black solid line, left inset).

erate state appears at zero energy for the $N_{I}=2$ notch. When the second notch is added in close proximity, only a single zero-energy state remains, according to the total sublattice imbalance of the system $N_{I}=2-1=1$.

In summary, defective structures with sublattice imbalance result in half-filled midgap states that are expected to yield magnetic moments when interactions are turned on. Structures with global sublattice balance can still present midgap states and be prone to developing local magnetic order, at least in two situations: distant defects with $N_{I}$ of opposite signs and large voids with sufficiently long zigzag edges.

\section{DEFECTS IN SEMICONDUCTING RIBBONS: INTERACTION EFFECTS}

In this section, we verify whether the physical picture anticipated from the noninteracting model remains true when the on-site Hubbard repulsions are included. As shown in Sec. IV, unpaired spins appear in sublattice imbalanced structures. When $\left|N_{I}\right|>1$, the noninteracting model predicts that a shell of $\left|N_{I}\right|$ degenerate midgap states is half-filled. The maximization of the spin is expected when Coulomb repulsions are turned on, in the spirit of Hund's rule. At halffilling, the exact ground state of the Hubbard model for a bipartite lattice such as that of graphene satisfies Lieb's ${ }^{33}$ theorem, which relates the sublattice imbalance and the ground state total spin: $2 S=\left|N_{I}\right|$. For unbalanced structures, this immediately confirms the Hund's rule scenario. In the case of balanced structures, the ground state spin must be zero, but this could happen with local moments, as it happens on the edges of infinite graphene zigzag ribbons.

The numerical calculations of this section are done with a unit cell of width $W$, length $L=N_{x} \frac{\sqrt{3}}{4} a$, where $N_{x}$ is the number of carbon atoms along an armchair chain, and with periodic boundary conditions along the $x$ direction to avoid spurious zigzag edges. We consider unit cells as long as $25 \mathrm{~nm}$ and the typical number of atoms in a self-consistent calcula-
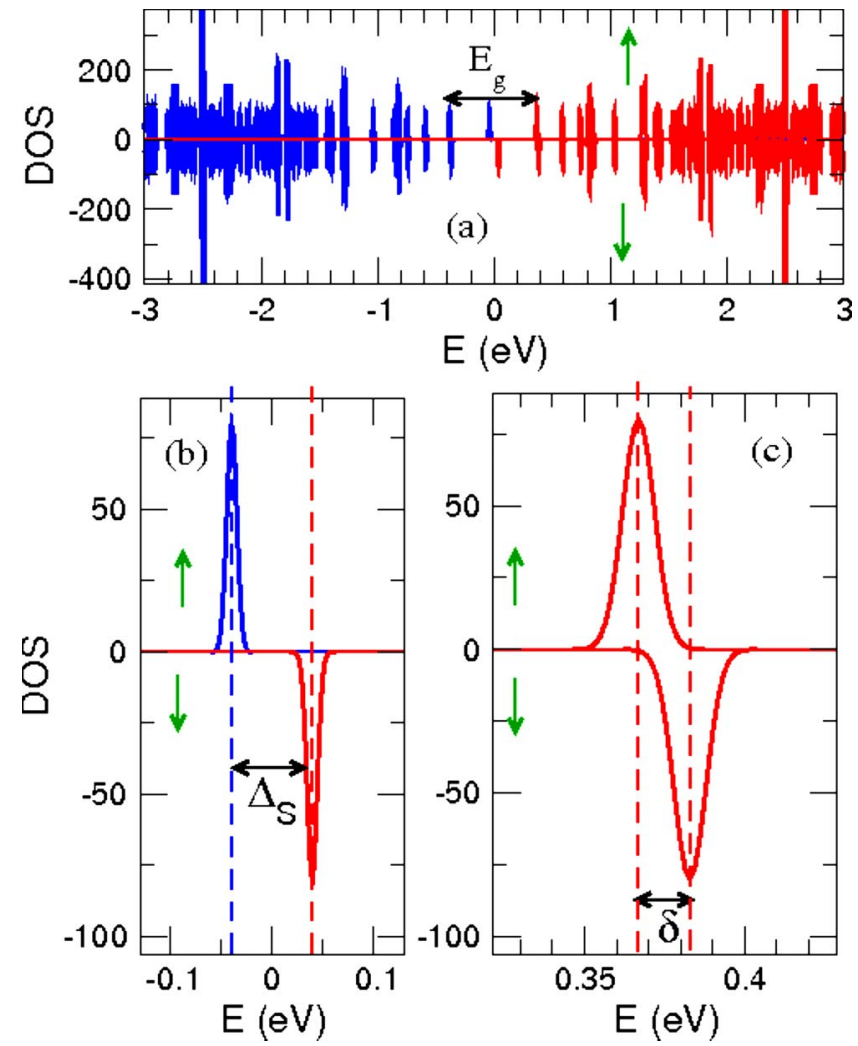

FIG. 6. (Color online) (a) The spin resolved DOS for a ribbon with $W=7 a$ and $U=2 \mathrm{eV}$ with one vacancy. Spin $\uparrow(\downarrow)$ is plotted as a positive (negative) number as a function of energy (we take the Fermi energy as zero). (b) Zoom of the spin-split midgap state. (c) Zoom of the conduction band minima. For clarity, we substitute the delta functions composing the DOS by Gaussian functions with a finite broadening.

tion is 1000. Importantly, our mean-field results have the same relation between the sublattice imbalance and ground state spin as the exact state, as predicted by Lieb's ${ }^{33}$ theorem.

\section{A. Single vacancy with $U \neq 0$}

We first revisit the single void samples. The ground state of structures with single-atom vacancies have one unpaired electron within the $U=0$ model and, according to Lieb's theorem, spin $1 / 2$ in the finite $U$ model. Our mean-field calculation for $U=2 \mathrm{eV}$ agrees with Lieb's ${ }^{33}$ theorem. There is a spin splitting of the midgap state $\Delta_{S}$ and a smaller spin splitting $\delta$ of the conduction and valence band states, as shown in Fig. 6. The spin degeneracy is thus broken, with only one of the spin channels of the midgap state occupied, the other being empty. This results in a finite magnetization density localized around the vacancy, as shown in Fig. 2. Although the magnetization mostly resides in the majority sublattice, interactions induce some reversed magnetization in the other sublattice.

\section{Analytical model}

We can gain some insight by doing an analytical description of the mean-field results that involves some approxima- 

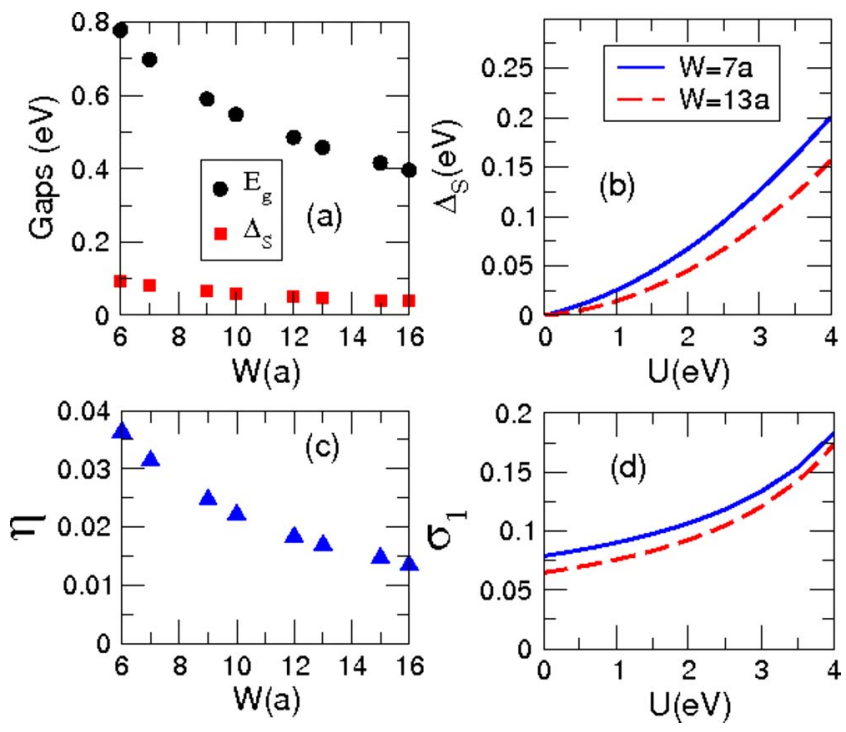

FIG. 7. (Color online) (a) Noninteracting $(U=0)$ energy gap (circles) and interacting $(U=2 \mathrm{eV})$ midgap spin splitting (squares) as a function of the ribbon width $W$. (b) Midgap spin splitting as a function of $U$ for two ribbon widths $W=7 a$ and $W=13 a$. (c) $U=0$ inverse participation ratio for the midgap state. (d) Standard deviation of the magnetization $\sigma_{1}$, as defined in Eq. (9), as a function of $U$ for two ribbon widths.

tions valid when $U$ is much smaller than the band gap of the ideal ribbon $E_{g}$. In this case, we assume that only the midgap state is spin polarized,

$$
\left\langle m_{i}\right\rangle_{0}=\frac{1}{2}\left|\phi_{v}(i)\right|^{2},
$$

where $\left|\phi_{v}(i)\right|^{2}$ is the $U=0$ square modulus of the midgap wave function. Notice that the normalization of $\phi_{v}(i)$ ensures that the total spin of the ground state is consistent with Lieb's ${ }^{33}$ theorem, $\Sigma_{i}\left\langle m_{i}\right\rangle_{0}=\frac{1}{2}$. The corresponding exchange splitting is

$$
\Delta_{S}=\epsilon_{0 \uparrow}-\epsilon_{0 \downarrow}=U \sum_{i}\left|\phi_{v}(i)\right|^{2}\left\langle m_{i}\right\rangle_{0}=\frac{U}{2} \sum_{i}\left|\phi_{v}(i)\right|^{4},
$$

where the sum is taken over all lattice sites.

We see that within the simplified analytical model, the spin splitting of the midgap state is proportional to the inverse participation ratio $\eta=\Sigma_{i}\left|\phi_{v}(i)\right|^{4}$. This quantity measures the degree of localization of the zero-energy state. An extended state in which the wave function is equally shared by $N$ atoms has $\eta=\frac{1}{N^{2}}$. In the opposite limit where the state is localized in a single atom, we would have $\eta=1$. The inverse participation ratio shown in Fig. 7(c) corresponds to a number of atoms in the range of $N=5$ to $N=9$. As discussed above, the localization of the midgap states plays an important role in the minimal distance at which they are effectively decoupled.

In Fig. 7(a), we plot the $U=0$ gap of the ideal ribbon $E_{g}$ and the $U=2 \mathrm{eV}$ spin splitting $\Delta_{S}$ of the midgap state as a function of the ribbon width $W$, as obtained from the full numerical calculation. As discussed above, we exclude the widths that give $E_{g}=0$. We see that the midgap spin splitting is a decreasing function of $W$. This is related to the fact that in the small $U$ limit, $\Delta_{S}$ is proportional to the inverse participation ratio $\eta$, which is also a decreasing function of the ribbon width, as shown in Fig. 7(c). The extension of the midgap state increases as the ribbon becomes wider, resulting in a reduction of the midgap spin splitting. As $E_{g}$ tends to zero (bulk graphene), the midgap state becomes non-normalizable ${ }^{46}$ and $\Delta_{S}$ is expected to vanish (see below).

Whereas the total magnetic moment $\Sigma_{i}\left\langle m_{i}\right\rangle$ is given by the sublattice imbalance, the degree of localization of the spin texture is not. In order to quantify it, we define the following standard deviation:

$$
\sigma_{1}=\sqrt{\sum_{i}\left\langle m_{i}\right\rangle^{2}}
$$

In this definition, $\sigma_{1}$ is not normalized as usual by $N$, the total number of atoms of the sample, since $\sigma_{1}$ characterizes a localized object. For sufficiently large simulation cells, doubling $N$ would imply a decrease of a normalized $\sigma_{1}$ without changing the local properties of the localized magnetic texture. Notice that within the analytical model valid for $U \ll E_{g}$, we have $\sigma_{1} \simeq \frac{1}{2} \sqrt{\eta}$. Hence, in the absence of staggered magnetization, both $\sigma_{1}$ and $\eta$ would measure the localization of the magnetic moments. For instance, if $\left\langle m_{i}\right\rangle$ $>0$ at all sites and $S=1 / 2$, the maximal $\sigma_{1}$ would be 0.5 . However, the graphene lattice responds with a staggered magnetization to the presence of defects and $\sigma_{1}$ also measures the magnitude of that response. In Figs. 7(b) and 7(d), we plot the midgap spin splitting $\Delta_{S}$ and $\sigma_{1}$ for two ribbons with $W=7 a$ and $W=13 a$ as a function of $U$. The midgap spin splittings can be fitted to $\Delta_{S}\left(N_{y}=7\right)(U)=0.0172 U$ $+0.0082 U^{2}$ and to $\Delta_{S}\left(N_{y}=13\right)(U)=0.0062 U+0.0082 U^{2}$. According to Eq. (8) the linear coefficients should be compared to $0.5 \eta$, which are 0.016 for $N_{y}=7$ and 0.008 for $N_{y}=13$. The nonlinear terms arise from the interaction-driven mixing between the midgap states and the conduction states. This is also consistent with the fact that $\sigma_{1}$ increases as a function of $U$, as shown in Fig. 7(d). The staggered magnetization is an increasing function of $U$. The coefficient of the quadratic term decreases with the length of the sample, namely, with the distance between vacancies since we are using periodic boundary conditions. We will come back to this issue in Sec. VII.

\section{Spin-charge separation for $U>0$}

We have verified that the ground states of structures with single-atom vacancies are locally neutral also with $U>0$ : the integrated electronic occupation in every site is one. Hence, a localized spin texture with total spin $1 / 2$ occurs in the absence of any charge localization. Our numerical results show that the addition of an extra electron to single vacancy structures results in a many electron state with total spin $S=0$, local magnetization which is zero everywhere, and local charge accumulated in the same atoms and with the same distribution as the magnetic texture of the charge neutral structure. These results are shown in Fig. 8 for a ribbon with $W=7 a$ and $U=2 \mathrm{eV}$. Hence, it is apparent that the mon- 

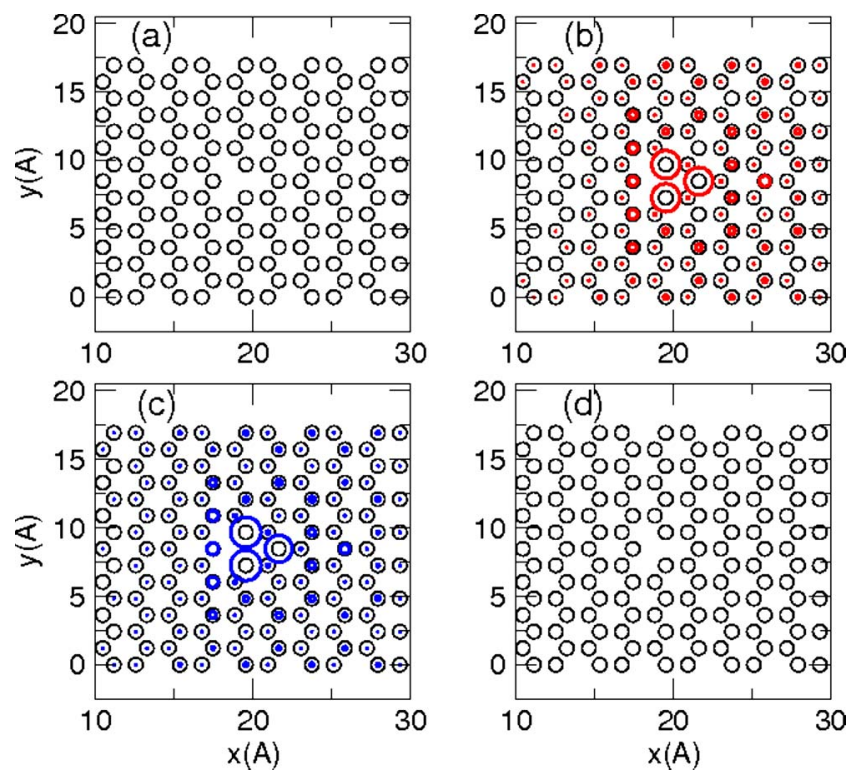

FIG. 8. (Color online) Spin-charge separation in single-atom vacancy. Left column: Neutral case. Right column: Charged case. Upper panels: Charge density $q_{i}-1$. Lower panels: $\left|\left\langle m_{i}\right\rangle\right| / \Sigma_{i}\left|\left\langle m_{i}\right\rangle\right|$. The local charge and local spin are zero everywhere for the neutral and charged cases, respectively. The spin texture of the neutral case is identical to the charge texture of the charged case.

atomic vacancy results in a multielectronic state with spincharge separation: the neutral ground state has a net electric charge $q=0$, but a total spin $S=1 / 2$ localized in a nonhomogeneous spin texture in locally neutral atoms. The charged ground state has a net charge $q=-1$, total spin $S=0$, no local magnetic moments, and a charge texture localized at the same location as the spin texture of the neutral ground state. This phenomenon resembles that reported by

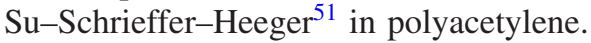

\section{Larger voids}

We have also calculated the mean-field magnetic structure for sublattice imbalanced larger voids. In Fig. 2(b), we show the magnetization profile for a triangular void with $N_{I}=2$. For the chosen value of $U=2 \mathrm{eV}$, the staggered magnetization is barely visible in this scale. In agreement with Lieb's ${ }^{33}$ theorem, it has a spin $S=1$ made out of local moments localized, mostly on the triangle boundaries. This object is the somehow complementary of the triangular graphene islands recently considered by Fernández-Rossier and Palacios. ${ }^{3}$ Figure 9 shows the ferrimagnetic spin texture around a rhomboidal void with imbalance charge $N_{I}=3-3=0$, i.e., composed of two triangular voids with $N_{I}= \pm 3$. Local moments with $\left\langle m_{i}\right\rangle \simeq 0.05$, three times smaller than those formed in the edges of infinite length zigzag ribbons, are formed on opposite corners of the void. We have verified that for $U=2 \mathrm{eV}$, the smallest void of this shape that features local moments is the one of the figure. The rhomboidal void is similar to the hexagonal islands considered in Ref. 3 in the sense that both have $S=0$ and develop local moments if they are sufficiently large.

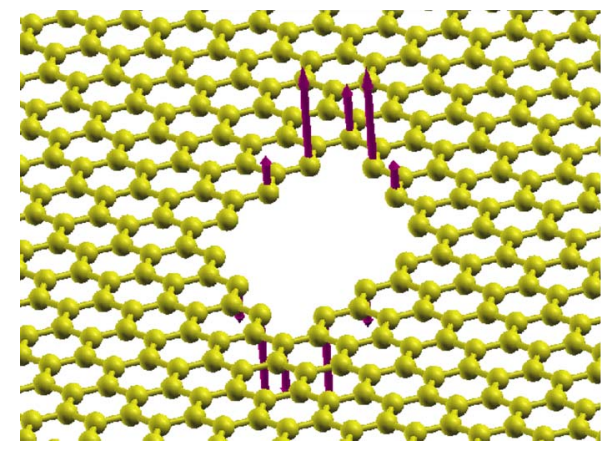

FIG. 9. (Color online) Emerging ferrimagnetic order in a rhombohedral void with imbalance charge $N_{I}=3-3=0$ situated in the middle of a ribbon with $W=10 a$ for $U=2 \mathrm{eV}$. The largest magnetic moment per atom is $\left\langle m_{i}\right\rangle=0.05$.

\section{B. Two vacancies with $U \neq 0$}

We now study the interaction between two magnetic defects with local sublattice imbalance $N_{I}= \pm 1$. Lieb's ${ }^{33}$ theorem warrants that when the sign of the sublattice imbalance is the same for the two defects, the total spin of the ground state is the sum of the spin of the individual defects. Hence, they are ferromagnetically coupled..$^{10,11,52}$ In contrast, if the two defects have opposite sublattice imbalance so that the global sublattice imbalance is zero, Lieb's ${ }^{33}$ theorem warrants that the total spin is zero. Our calculations show that this can happen in two different scenarios: the local magnetization might be zero everywhere or the two defects could be magnetized along opposite directions, i.e., could be antiferromagnetically coupled. When the defects are sufficiently far apart from each other, their local electronic structure should be identical to that of single defects. Hence, the spin interaction between two magnetic defects can be either ferromagnetic or antiferromagnetic, as in the case of indirect exchange interactions (RKKY) between single site magnetic moments, ${ }^{53,54}$ but can also result in the annihilation of the local magnetic order, a scenario that goes beyond the RKKY picture.

In Fig. 10, we plot the normalized standard deviation of the two magnetic moments $\sigma_{2}$ for ribbons with $W=7 a$ and $W=13 a$ as a function of the defect separation. We normalize the computed $\sigma_{2}$ to the one corresponding to two independent single-defect magnetic textures, $\sqrt{2} \sigma_{1}$. When the defects are sufficiently far away, $\sigma_{2}$ must tend to $\sqrt{2} \sigma_{1}$, i.e., the normalized $\sigma_{2}$ must tend to 1 . We consider the effect of the ribbon width $W$, interaction strength $U$, and sublattice imbalance upon the magnetic interactions between the two defects. In the case of $W=7 a$, we show results for both monatomic vacancies lying on the same sublattice $(A+A$, open circles), whose ground state total spin is $S=1$, and on different sublattices $(A+B$, full circles), whose ground state spin is $S=0$. The two curves for $W=7 a$ are calculated with $U=2 \mathrm{eV}$. At large distances, the two defects become decoupled, as expected. At short distances, the behavior of the magnetic texture is radically different for both $A+A$ and $A+B$ structures. In the former case, $\sigma_{2}$ is enhanced, indicating the localization of the magnetic texture in a smaller region. Since the total spin is 1 , local moments survive even when the two defects 


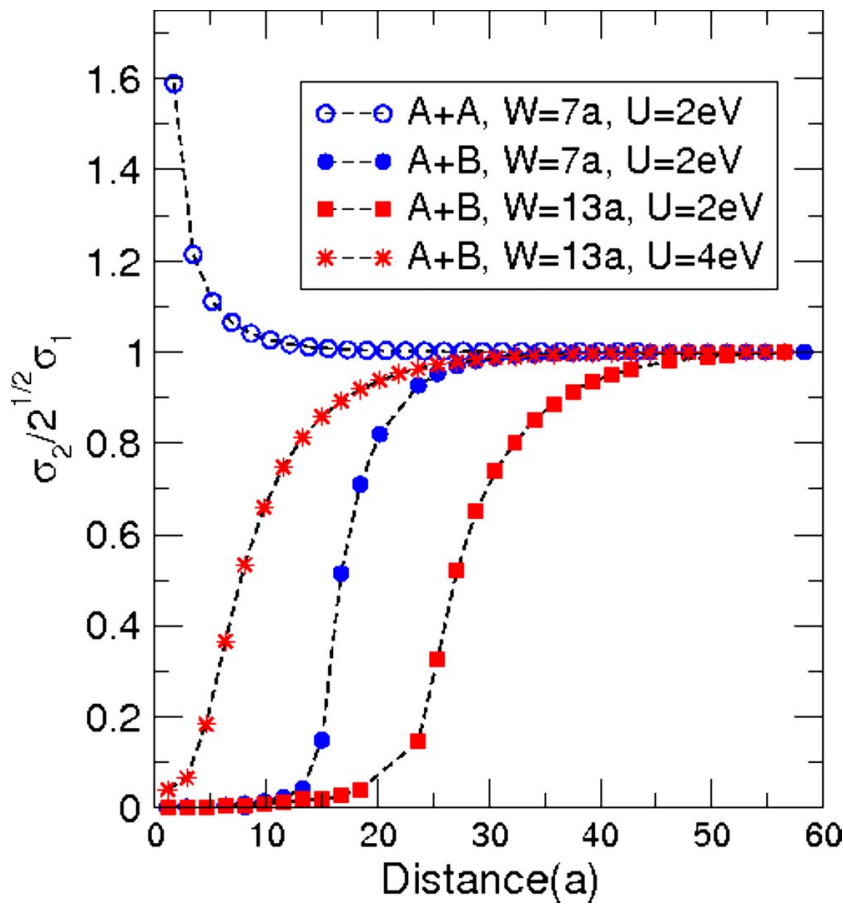

FIG. 10. (Color online) Normalized standard deviation of the magnetization as a function of the distance between vacancies for four cases: ribbon with $W=7 a$ and $U=2 \mathrm{eV}$, vacancies in the same sublattice (open circles); same ribbon but vacancies in different sublattices (full circles); ribbon with $W=13 a$, vacancies in different sublattices (full squares); same as the previous case but with $U$ $=4 \mathrm{eV}$ (stars).

are very close. As the separation between defects increases, they become independent from each other and $\sigma_{2}=\sqrt{2} \sigma_{1}$. When this happens, the energy gap between $S=1$ and $S=0$ should vanish. This is an example of rule (1).

In contrast to the $A+A$ case, the local magnetization of the $A+B$ structure vanishes below a minimal distance $D_{c}$. This is an important result. In other words, there is a maximal density of defects above which zero-energy states hybridize and local magnetic moments vanish. The critical density depends on the energy scales of the problem, the singleparticle gap $E_{g}$, controlled by the ribbon width, and the onsite repulsion $U$. For fixed $U$, the decoupling distance is definitely shorter for $W=7 a$ than for $W=13 a$. Hence, the critical (linear) density becomes smaller as the ribbon width increases. This is consistent with the fact that both the $U$ $=0$ inverse participation ratio and the $U \neq 0 \quad \sigma_{1}$ are decreasing functions of the ribbon width $W$. The wider the ribbon, the larger the delocalization of the zero-energy state. Hence, the hybridization between the midgap states associated with each vacancy survives at a larger distance for wider ribbons. Finally, in Fig. 10, we also show $\sigma_{2}$ for $W=13 a$ and two values of $U, 2$ and $4 \mathrm{eV}$. The decoupling distance (critical density) decreases (increases) as a function of $U$. In other words, interactions drive the system magnetic, as expected. The $A+B$ case is the simplest example that exemplifies rules (2) and (3).

According to the two-vacancy calculation shown in Fig. 10, the magnetic (low density, large intervacancy distance) and nonmagnetic (high density, low intervacancy distance) phases are separated by a crossover region. If we take as an estimate of the critical distance below which local moments are quenched the distance for which $\sigma_{2}\left(D_{c}\right) / \sqrt{2} \sigma_{1}=0.5$, we find that $D_{c}=40 \AA$ for $W=7 a$ and $D_{c}=65 \AA$ for $W=7 a$, both for $U=2 \mathrm{eV}$. The corresponding critical linear densities $n_{c} \equiv \frac{1}{D_{c}}$ are $n_{1 c}(W=7 a)=2.5 \times 10^{6} \mathrm{~cm}^{-1}$ and $n_{1 c}(W=13 a)$ $=1.5 \times 10^{6} \mathrm{~cm}^{-1}$, respectively. The corresponding areal densities $n_{2 c}=\frac{1}{W \times D_{c}}$ are $n_{2 c}(W=7 a)=1.4 \times 10^{13} \mathrm{~cm}^{-2}$ and $n_{2 c}(W=13 a)=4.8 \times 10^{12} \mathrm{~cm}^{-2}$, respectively. These numbers should be taken as order of magnitude estimates of the real critical density.

In the case of $A+B$ pairs, the crossover from the locally magnetic to the nonmagnetic state is similar to the one described in compensated graphene nanoislands: ${ }^{3}$ small islands are nonmagnetic and larger islands have magnetic edges. The critical density depends on the extension of the magnetization, which, in turn, depends on the ribbon single-particle gap $E_{g}$ (which controls the extension of the $U=0$ midgap states) and on the on-site repulsion $U$. The quenching of the local moments in the $A+B$ structures is definitely related to the hybridization of the midgap states described in the noninteracting model. This phenomenon has an analog in zigzag ribbons. The midgap bands are linear combinations of top and bottom edge states. The hybridization is negligible in the Brillouin zone boundary and is much larger in the middle. As a result, the exchange interaction strongly renormalizes the zone-boundary states, opening a magnetic gap, but they barely change in the middle of the zone. ${ }^{38}$

\section{Defective graphene ribbons as diluted magnetic semiconductors}

The physical picture that emerges from the previous discussion leads to an interesting conclusion: A semiconducting graphene ribbon with a density of vacancies that induce magnetism will behave like a diluted (para)magnetic semiconductor ${ }^{55}$ (DMS) provided that the density of defects is smaller than the critical density defined above [this would be an example of rule (3)]. Charged excitations will present a gap and spin excitations will not. The long range ferromagnetic order found by Pisani et al. ${ }^{52}$ only occurs when the vacancies are all in the same sublattice. It remains an open issue whether or not such a sublattice imbalance might occur in reality. Unless this can be shown, one should not expect long range ferromagnetic order in real samples.

Interestingly, the conduction and valence bands depend on the magnetic order of the local moments, which might be induced by application of an external magnetic field. In the DMS case, the conduction and valence bands are exchanged coupled to the local moments provided by Mn atoms. At zero field, the Mn spins are randomly oriented and the average spin splitting of the bands is zero. Application of an external field orders the Mn spins, resulting in a finite average exchange-induced spin splitting of the bands, which is much larger than the standard Zeeman splitting. This is known as the giant Zeeman splitting.

The same scenario might occur in semiconducting graphene ribbons with magnetic defects. When the interde- 

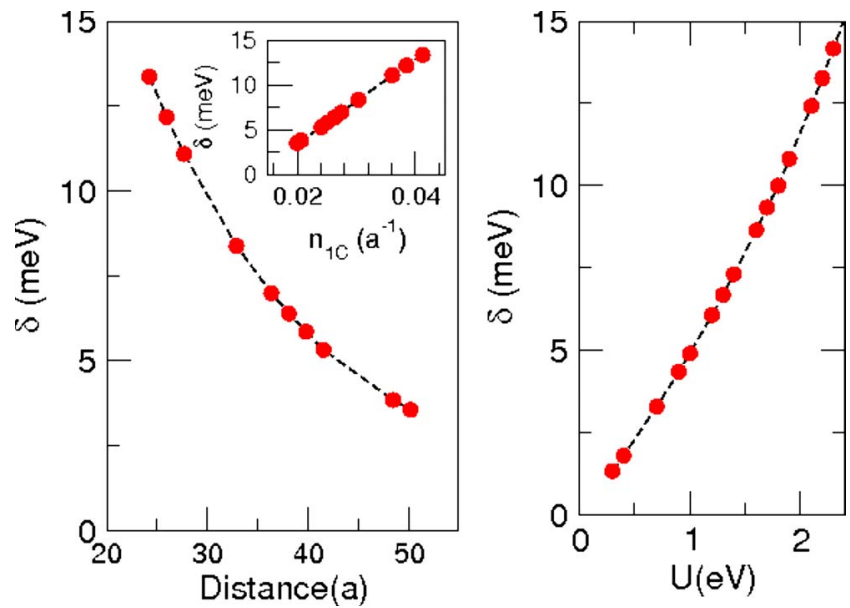

FIG. 11. (Color online) Left panel: Bottom of the conduction band spin splitting $\delta$ as a function of the vacancy distance and as a function of the linear vacancy density (inset) for a ribbon with $W$ $=7 a$ and $U=2 \mathrm{eV}$. Right panel: $\delta$ for the same ribbon for a fixed vacancy distance of $28 a$ as a function of $U$.

fect distance is larger than the critical spacing $D_{c}$, the local moments become independent. Application of a magnetic field aligns them and induces an exchange-induced splitting of the conduction and valence bands much larger than the intrinsic Zeeman splitting. ${ }^{55}$ In the case of graphene ribbons with vacancies, we have computed the spin splitting of the bottom of the conduction band,

$$
\delta \equiv E_{\uparrow}-E_{\downarrow},
$$

where $E_{\sigma}$ is the first level above the midgap state [see Fig. 6(c)]. Notice that the shift of the top of the valence band and the bottom of the conduction band is such that the gap, ignoring the midgap states, is spin independent. Since we consider independent vacancies, the calculation is done with a single vacancy per unit cell. In Fig. 11, we plot $\delta$ for a ribbon with $W=7 a$. In the left panel, we plot $\delta$ as a function of the interdefect distance considering only values bigger than $D_{c}$ for which the defects are decoupled. For a $U=2 \mathrm{eV}$, we find that $\delta$ ranges between 5 and $15 \mathrm{meV}$ for the range of distances considered. This splitting could be obtained with an applied magnetic field such that $g \mu_{B} B \gg k T$; yet, $\delta \gg g \mu_{B} B$. As in the case of real DMS, this giant Zeeman splitting linearly scales with the defect density, as shown in the inset of the right panel of Fig. 11. Since there is a maximal density above which the local moments are coupled and eventually they vanish, the splitting $\delta$ cannot be indefinitely increased. This phenomenon also has an analog in DMS: direct antiferromagnetic coupling between $\mathrm{Mn}$ spins eventually blocks the paramagnetic coupling to the external field.

In the right panel of Fig. 11, we plot $\delta$ as a function of $U$. For small $U$, we find that $\delta$ is almost linear with $U$. This can be understood in the framework of the analytical model discussed above. If we consider that, to lowest order, the magnetization only comes from the midgap states with wave function $\phi_{v}(i)$ and we compute the splitting of the bottom of the conduction band states to first order perturbation theory, we obtain

$$
\delta=U \sum_{i}\left|\phi_{c}(i)\right|^{2} \sum_{v}\left|\phi_{v}(i)\right|^{2},
$$

where $\phi_{c}(i)$ is the $U=0$ single-particle state of the bottom of the conduction band and the summation is taken over lattice sites. If we approximate $\phi_{c} \simeq 1 / \sqrt{N}$, where $N$ is the number of atoms in the unit cell, and we use the normalization condition of the midgap states, $\Sigma_{i}\left|\phi_{v}(i)\right|^{2}=1$, then we have

$$
\delta \simeq \frac{U N_{v}}{N}
$$

where $N_{v}$ is the number of magnetic vacancies per unit cell. This equation accounts also for the fact that $\delta$ linearly scales with the defect density. Deviations from the linear behavior arise due to the magnetization that arises from states other than midgap states.

The strong sensitivity of the conduction states of the defective armchair ribbon on the application of a moderate magnetic field should give rise to strong spin-dependent magnetotransport and magneto-optic effects, in analogy with DMS spintronic devices. Notice that in contrast to standard Mn doped II-VI semiconductors, for which electrical injection of carriers results in a new carrier mediated coupling, ${ }^{56,57}$ the addition of carriers in this system would result in the compensation of the midgap states and the disappearance of the local moments, as shown in Fig. 8.

\section{DEFECTS IN BULK GRAPHENE: VACANCIES}

So far, we have considered the electronic structure of semiconducting graphene ribbons with vacancies and voids. As shown in Fig. 10, the critical distance for the quenching of the magnetic moments increases with the ribbon width. An important question is whether or not this critical distance converges to a finite value in the two-dimensional limit. We have also seen in Fig. 7(d) how the (standard deviation of the) magnetization $\sigma_{1}$ associated with vacancies decreases as the gap of the ribbon decreases. In this section, we address the question of what happens to these and other results obtained above in the limit of infinitely wide ribbons where the gap goes to zero, i.e., bulk graphene. The extrapolation to the two-dimensional case is not straightforward. We thus consider a different strategy. Here, we consider unit cells with periodic boundary conditions in both directions. An infinite graphene crystal with a unit cell formed by $N_{y}$ parallel armchairlike chains, each of them containing $N_{x}$ carbon atoms. The dimension of this unit cell is $\left(N_{x} \frac{\sqrt{3}}{4} a, N_{y} a\right)$, where $a$ is the graphene lattice parameter. We are interested in square unit cells and, therefore, we consider units cells $\left(N_{x}, N_{y}\right)$ with sizes of $(24,10),(32,13),(40,16),(48,20),(60,26)$, and $(72,31)$. We locate one or more vacancies in the unit cell considered and, as for ribbons, we obtain the eigenvalues, eigenfunctions, and the magnetization at each place of the system, $\langle m(i)\rangle$, by solving self-consistently the Hamiltonian.

\section{A. System with vacancies of the same type}

We locate a vacancy at the center of the unit cell in such a way that our system describes a square lattice of vacancies 


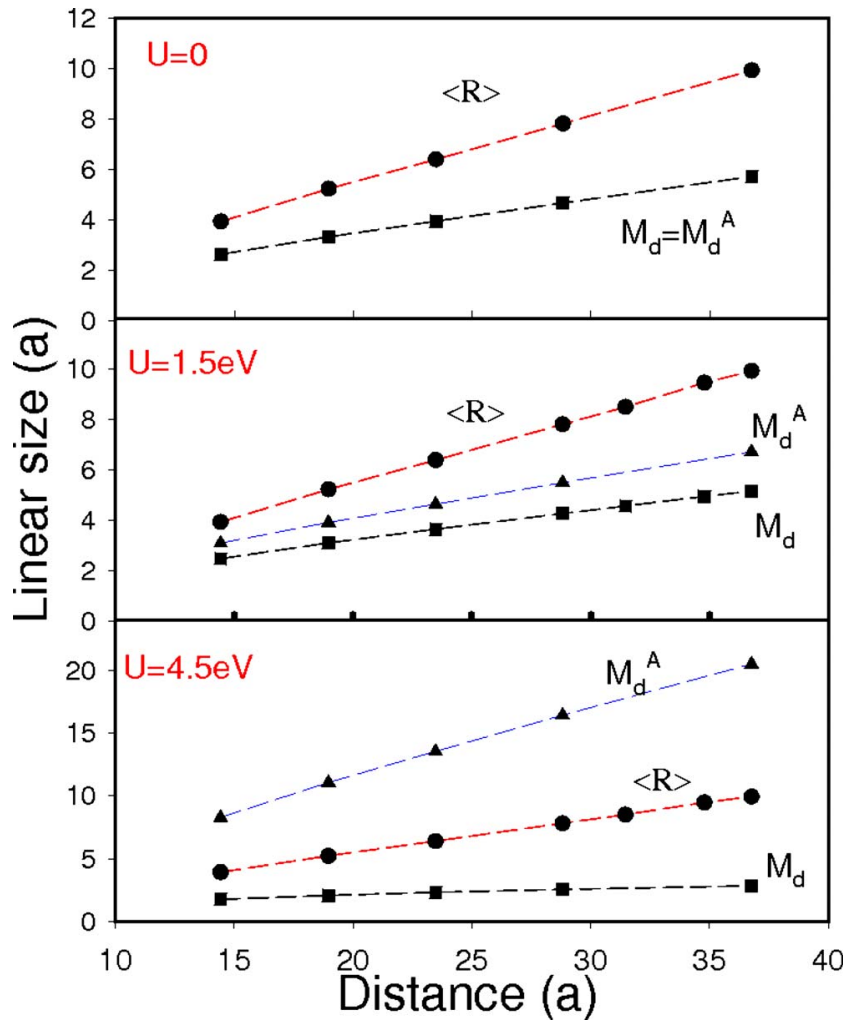

FIG. 12. (Color online) Linear size of the wave function of the vacancy $\langle R\rangle$ of the net magnetization $M_{d}$ and of magnetization in the sublattice $A, M_{d}^{A}$, as function of the distance between vacancies. From top to bottom, the panels correspond to Hubbard constants, $U=0, U=1.5$, and $U=4.5 \mathrm{eV}$. The vacancies are located in site type $B$.

all located on the same type of atoms, e.g., $B$ in our notation. We obtain that in agreement with Lieb's ${ }^{33}$ theorem, the ground state of the system has a magnetic moment $S=1 / 2$ per vacancy. We also obtain the spin gap of the system $\Delta_{S}$, the wave function $\phi_{v}(i)$ of the first empty state, and the local magnetization. In the limit of a large unit cell, $\Delta_{S} / 2$ and $\phi_{v}(i)$ should be the energy and the wave function, respectively, of an isolated vacancy. In two dimensions, we can characterize the linear size of the vacancy wave function by its first moment

$$
\langle R\rangle=\sum_{i}\left|\mathbf{r}_{i}-\mathbf{r}_{0} \| \phi_{v}(i)\right|^{2}
$$

where $\mathbf{r}_{0}$ is the position of the vacancy and the sum is over all atoms of the unit cell. As discussed above, the bipartite character of the graphene lattice produces an antiferromagnetic coupling between the magnetization of the two sublattices of the system. ${ }^{53,54}$ We define the linear size of the magnetization in each sublattice as

$$
M_{d}^{A(B)}=\sum_{i \in A(B)}\left|\mathbf{r}_{i}-\mathbf{r}_{0}\right|\left\langle m_{i}\right\rangle .
$$

$M_{d}^{A}$ and $M_{d}^{B}$ have opposite signs, and their sum, $M_{d}=M_{d}^{A}$ $+M_{d}^{B}$, indicates the extension of the net magnetization.

In Fig. 12, we plot the linear size of the vacancy wave function and of the magnetization as function of the distance between vacancies for different values of $U$. The first thing to note is that the size of the wave function linearly increases with the size of the unit cell, which is practically independent of the value of $U$. This result indicates that the electronelectron interaction almost does not affect $\phi_{v}$, which, when $U=0$, becomes a quasilocalized state with weight in only one sublattice $(A)$ and decays as $1 / r$, in agreement with analytical results. ${ }^{46}$ When $U=0$, only sublattice $A$ is magnetized and $M_{d}=M_{d}^{A}$. However, $M_{d}$ is considerably smaller than $\langle R\rangle$, indicating that the presence of the vacancy does not just create a quasilocalized state but also strongly modifies the wave functions of the states in the continuum. Notice the difference with the ribbons where the magnetization follows the wave function for sizable confinement gaps.

As we increase $U$, the magnetic texture evolves in such a way that their size $M_{d}$, as defined in Eq. (14), decreases. This is accompanied by an increase of the staggered magnetization, reflecting the antiferromagnetic tendency of the bipartite lattice, which polarizes sublattice $B$ in the opposite direction as that of sublattice $A$ with $M_{d}^{A}>M_{d}$. This effect can be rather dramatic for moderate values of $U$; in Fig. 12 we show that for $U=4.5 \mathrm{eV}$, the extension of the magnetization in sublattice $A$ is considerably larger than $\langle R\rangle$ and $M_{d}$. Note that $U=4.5 \mathrm{eV}$ is still below the critical value $U_{A F}$ $\sim 5.5 \mathrm{eV}$ for the occurrence of an antiferromagnetic instability in perfect graphene..$^{10,58,59}$ Thus, a network of vacancies in the same sublattice would have a magnetic ground state, which is in agreement with Lieb's ${ }^{33}$ theorem, and enhanced staggered magnetization compared to perfect graphene.

We now consider the midgap spin splitting $\Delta_{S}$ in twodimensional graphene with a finite density of vacancies in the same sublattice. In the previous case of semiconducting ribbons, there was a strong indication that $E_{g}>\Delta_{S}$ for any ribbon width in the single vacancy limit. Hence, we might expect that $\Delta_{S}$ vanishes in two-dimensional graphene. When we have a finite density of defects, $\Delta_{S}$ has also an interdefect contribution arising from the hopping term. This mechanism is possible only if the midgap states have weight on the two sublattices. Midgap states associated with defects in the same sublattice can only be coupled through interaction-driven sublattice mixing. As the gap is a product of the coupling between the magnetizations induced by the Hubbard interaction, the value of the gap quadratically increases with $U$ to lowest order. Our calculations in Fig. 13(a) show that for $U \neq 0$, the midgap spin splitting $\Delta_{S}$ goes to zero as the density of vacancies goes to zero. In Fig. 13(b), we plot the weight of $\phi_{v}(i)$ on the sublattice where the vacancy is located. This quantity also tends to zero as the density of defects decreases. Thus, in the single impurity limit, the spin gap goes to zero and the interacting $\phi_{v}(i)$ lives only on one sublattice.

\section{B. Vacancies on different sublattices: Compensated case}

In principle, one could expect that in real graphene samples, the number of vacancies on sublattices $A$ and $B$ are roughly equal. In this situation, Lieb's ${ }^{33}$ theorem requires 


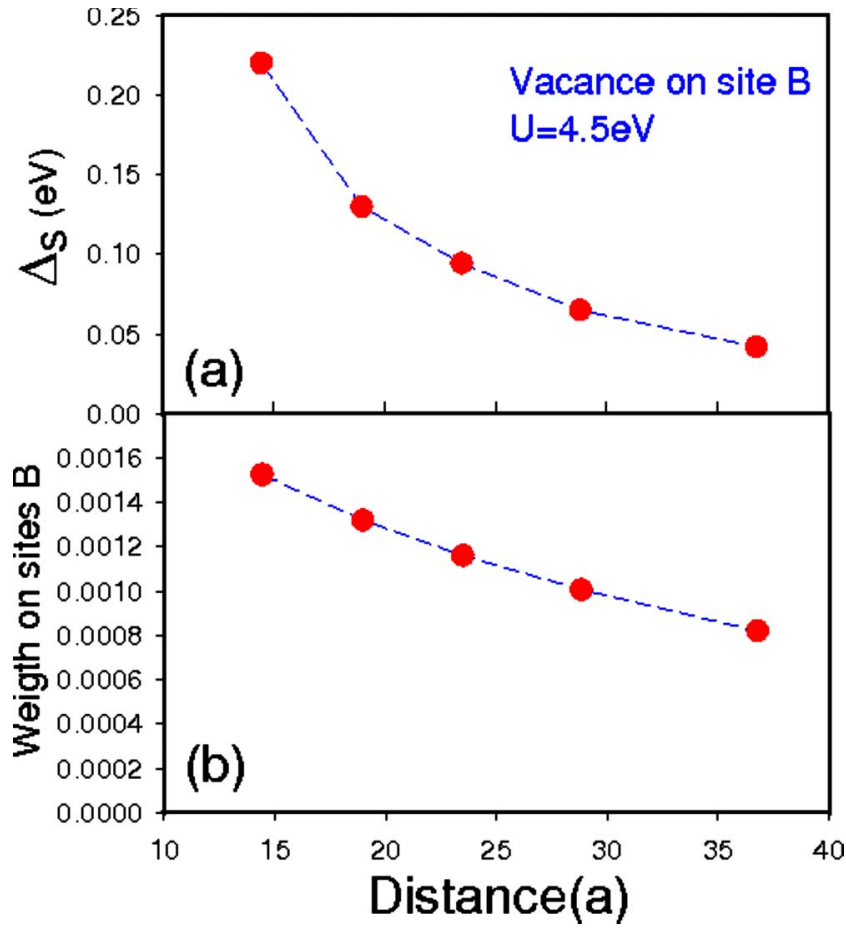

FIG. 13. (Color online) (a) Midgap spin splitting as function of the distance between vacancies and (b) weight of the quasilocalized wave function on site $B$. The vacancies are located in the sublattice $B$ and $U=4.5 \mathrm{eV}$.

that the total spin of the system should be essentially zero. From the results in the case of ribbons, one should expect an antiferromagnetic coupling between the magnetic moments for small concentrations of vacancies. For large concentrations, the local magnetic moments should disappear and the sample should turn nonmagnetic. In order to study the interaction between vacancies located in different sublattices and the local magnetization in a compensated system, we locate two vacancies with total sublattice imbalance equal to zero in the unit cell. The $A$ and $B$ vacancies form two interpenetrated square lattices in such a way that the distance between impurities is maximum. We have checked that in agreement with Lieb's ${ }^{33}$ theorem, the $S=0$ solution is the ground state of the system.

We quantify the local magnetization studying the standard deviation of $\left\langle m_{i}\right\rangle$,

$$
\sigma=\sqrt{\frac{\sum_{i}\left\langle m_{i}\right\rangle^{2}}{N}},
$$

where the sum is over all carbon atoms and $N=N_{x} \times N_{y}$ is the number of atoms in the unit cell. In the inset of Fig. 14, we plot $\sigma$ as a function of $U$ for a unit cell with size of $(26,60)$, which corresponds to a density of vacancies of 0.5 $\times 10^{13} \mathrm{~cm}^{-2}$. We obtain that for small values of $U$, the magnetization is zero everywhere and that there is a critical value of the Hubbard coupling $U_{c}$, for which a local magnetization near the vacancies appears. ${ }^{10}$ This critical $U$ depends on the density of vacancies, and in Fig. 14, we plot $U_{c}$ as function of the density of vacancies. We obtain that $U_{c}$ decreases with

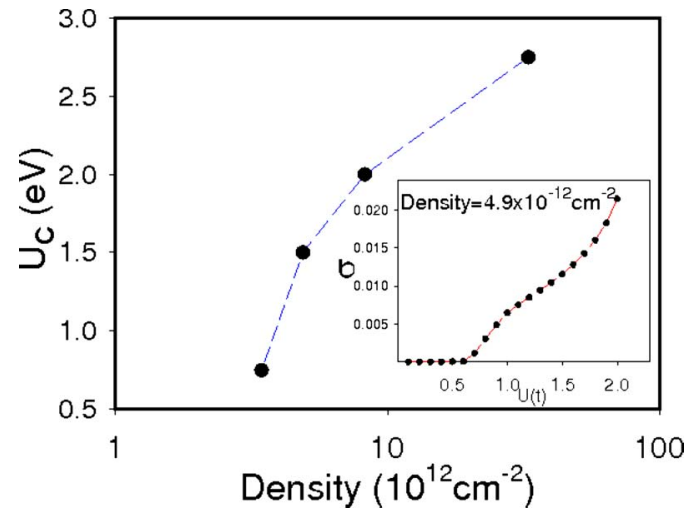

FIG. 14. (Color online) Critical values for the occurrence of a magnetic texture in a system of vacancies on atoms of types $A$ and $B$ forming two square interpenetrated lattices. The inset indicates the variation of the standard deviation of the local magnetizations as a function of $U$ for a system with dimension of $(26,60)$.

the density of vacancies and from our results we conclude that in the limit of zero density, $U_{c}$ tends to zero. In this limit, each vacancy hosts a spin $1 / 2$ texture, which is decoupled from the others. For high density of vacancies and moderate values of $U$, the kinetic energy coupling between the vacancies is stronger than the electronic repulsion, and in order to minimize the energy, the system makes the local magnetization zero everywhere. When the density of impurities decreases, the kinetic energy coupling between vacancies decreases and eventually the impurities become uncoupled and each of them gets a total spin $\pm 1 / 2$ and the system behaves as a diluted antiferromagnetic system. ${ }^{53} \mathrm{Un}$ less a reason for global lattice imbalance exists (unknown to us), for realistic values of $U$ in the range $1<U<2 \mathrm{eV},{ }^{3}$ we expect that in highly irradiated graphene samples, the local magnetization should be zero across the sample.

\section{DISCUSSION AND CONCLUSIONS}

Some of the possible limitations of our approach have already been mentioned in Sec. II. We have left aside the issue of the structural stability of passivated vacancies and voids. Away from the edges, a single monatomic vacancy might not result in a local atomic configuration that can be described with our model. On the other side, the effect of a hydrogen atom atop a carbon atom on graphene is very similar to the one described by our model. ${ }^{11}$ Hence, the anomalous magnetic behavior of irradiated graphite might be related to $H-C$ pairs rather than to missing atoms. It is also important to signal that the mean-field approximation is known to overestimate the appearance of magnetic order and yield critical values of $U / t$ smaller than those obtained with methods that include quantum fluctuations. ${ }^{58}$ Finally, we have neglected both second neighbor hopping and interatomic Coulomb repulsions. Interestingly, both DFT and mean-field Hubbard models yield a very similar description of the magnetic behavior of graphene islands ${ }^{3}$ and zigzag graphene ribbons. ${ }^{38}$ This indicates that the couplings neglected in the simple Hubbard model have a small effect on 
the low-energy electronic structure that dominates the physical properties.

We now summarize the main conclusions of this work. In the context of our model, the main results are as follows.

(1) The electronic properties of the defects arising from the removal of atoms from graphene dramatically depend on an integer number, the sublattice imbalance (or imbalance charge) $N_{I}=N_{A}-N_{B}$, which counts the difference in the total number of atoms per sublattice removed from a perfectly balanced graphene lattice. $N_{I}$ can take values $0, \pm 1, \pm 2$, etc.

(2) It can be rigorously shown ${ }^{29}$ that the single-particle spectrum of a structure with sublattice imbalance $N_{I}$ has, at least, $\left|N_{I}\right|$ midgap states per spin channel occupied by $\left|N_{I}\right|$ electrons in neutral graphene.

(3) Repulsive Coulomb interactions will result in a manybody ground state with $2 S=\left|N_{I}\right|$. This is an exact result in the case of the Hubbard model. ${ }^{33}$

(4) Whereas the total magnetic moment of a given graphene system is given by Lieb' ${ }^{33}$ theorem, the local magnetic structure is not. By assigning local sublattice imbalance numbers to defects, provided that they are sufficiently apart, a set of rules to predict basic features of the magnetic structure has been proposed.

(5) We find that single voids with $\left|N_{I}=1\right|$ give rise to states with spin-charge separation, in the sense that a localized magnetic texture does not entail charge localization. The addition of a single electron to the system results in a manybody state with $S=0$ and the disappearance of the magnetic texture, which is substituted by a charge texture, as seen in Fig. 8. In this sense, the properties of these states are very similar to Su-Schrieffer-Heeger midgap states. ${ }^{51}$

(6) The addition rules for two voids with a given local sublattice imbalance or imbalance charge present similarities with those of vortices, e.g., in superconductors. When sufficiently apart, two voids with local imbalance $+N_{I}$ and $-N_{I}$ behave like two independent objects with local spin $2 S$ $=\left|N_{I}\right|$. Below a certain distance, they annihilate each other and the local magnetization vanishes (Fig. 10). When two voids with the same sign are brought together, they result in a region with enhanced local magnetization and spin $2 S$ $=\left|N_{I}\right|+\left|N_{I}\right|$, as seen in Fig. 2 .

(7) In analogy with graphene nanoislands, ${ }^{3}$ sufficiently large voids with $N_{I}=0$ can still have local magnetic moments. These can interpreted as if the large void with $N_{I}=0$ was the sum of two voids with $\pm N_{I}^{\prime}$. An example of this is the rhomboid of Fig. 9 obtained from merging two triangular voids with $N_{I}= \pm 3$ back to back.

(8) Our results show that spin interactions between two magnetic defects of same $\left|N_{I}\right|$ can be of three types: ferro- magnetic and antiferromagnetic or annihilating. In the first case, the ground state spin is the sum of the spins of the magnetic defects when infinitely apart. In the second case, the spin is the difference between those two. In the third case, both the total and the local spins are zero. Antiferromagnetic and annihilating couplings occur in lattices without global sublattice imbalance, whereas ferromagnetic coupling requires global sublattice imbalance.

(9) Our simulations show that in balanced defective structures, there is a maximal density of monatomic vacancies that can sustain local moments. In the case of ribbons, this critical density depends on the ribbon width. The critical density also depends on $U$. A phase diagram for bulk graphene is provided in Fig. 14.

(10) Depending on the density of vacancies, distributed randomly in the two sublattices, we distinguish three phases. In the very dilute limit, the system is paramagnetic with some common properties with II-VI diluted magnetic semiconductors. In the opposite high-density limit, the local moments are annihilated. The intermediate phase features antiferromagnetically coupled local moments. This would be a realization of the so-called diluted antiferromagnet. ${ }^{53}$

(11) We predict giant Zeeman splitting in the case of semiconducting ribbons in the dilute limit. Upon application of an external magnetic field such that $g \mu_{B} H>k_{B} T$, the magnetic moment of all the defects would point parallel to the applied field. This would result in an interaction induced splitting in the band states, much larger than the ordinary Zeeman splitting, as seen in Fig. 11.

(12) A ferromagnetic phase is not expected for defective graphene, unless vacancies predominantly occur in one of the two sublattices. Such an unbalanced situation would require further explanation.

(13) In the case of zero-gap graphene, we find that midgap states survive even in the interacting case, in the very dilute limit (Fig. 13). Since ideal graphene is a semimetal, the thermodynamic properties of graphene might be dominated by this type of defects.

Note added. Recently, a related work by Yazyev $^{60}$ has been reported.

\section{ACKNOWLEDGMENTS}

We acknowledge fruitful discussions with F. Guinea, B. Korgel, and P. López-Sancho. This work was financially supported by MEC-Spain under Grant Nos. MAT2007-65487, MAT2006-03741, and CONSOLIDER CSD2007-00010, and by Generalitat Valenciana under Grant No. ACOMP07/054.

\footnotetext{
${ }^{1}$ J. M. D. Coey, Solid State Sci. 7, 660 (2005).

${ }^{2}$ H. Ohldag, T. Tyliszczak, R. Hohne, D. Spemann, P. Esquinazi, M. Ungureanu, and T. Butz, Phys. Rev. Lett. 98, 187204 (2007).

${ }^{3}$ J. Fernández-Rossier and J. J. Palacios Phys. Rev. Lett. 99, 177204 (2007).

${ }^{4}$ R. C. Longo and L. J. Gallego, Phys. Rev. B 74, 193409 (2006).
}

\footnotetext{
${ }^{5}$ W. Luo, S. J. Pennycook, and S. T. Pantelides, Nano Lett. 7, 3134 (2007).

${ }^{6}$ P. Crespo, R. Litrán, T. C. Rojas, M. Multigner, J. M. de la Fuente, J. C. Sánchez-López, M. A. García, A. Hernando, S. Penadés, and A. Fernández, Phys. Rev. Lett. 93, 087204 (2004).

${ }^{7}$ Y. Yamamoto, T. Miura, M. Suzuki, N. Kawamura, H. Miya-
} 
gawa, T. Nakamura, K. Kobayashi, T. Teranishi, and H. Hori, Phys. Rev. Lett. 93, 116801 (2004).

${ }^{8}$ P. O. Lehtinen, A. S. Foster, Y. Ma, A. V. Krasheninnikov, and R. M. Nieminen, Phys. Rev. Lett. 93, 187202 (2004).

${ }^{9}$ M. A. H. Vozmediano, M. P. Lopez-Sancho, T. Stauber, and F. Guinea, Phys. Rev. B 72, 155121 (2005).

${ }^{10}$ H. Kumazaki and D. S. Hirashima, J. Phys. Soc. Jpn. 76, 064713 (2007).

${ }^{11}$ Oleg V. Yazyev and Lothar Helm, Phys. Rev. B 75, 125408 (2007).

${ }^{12}$ T. Chanier, I. Opahle, M. Sargolzaei, R. Hayn, and M. Lannoo, Phys. Rev. Lett. 100, 026405 (2008).

${ }^{13}$ M. Ezawa, Phys. Rev. B 76, 245415 (2007); M. Ezawa, Physica E (Amsterdam) 40, 1421 (2008).

${ }^{14}$ K. S. Novoselov, A. K. Geim, S. V. Morozov, D. Jiang, Y. Zhang, S. V. Dubonos, I. V. Grigorieva, and A. A. Firsov, Science 306, 666 (2004).

${ }^{15}$ J. S. Bunch, Y. Yaish, M. Brink, K. Bolotin, and P. L. McEuen, Nano Lett. 5, 287 (2005).

${ }^{16}$ K. S. Novoselov, A. K. Geim, S. V. Morozov, D. Jiang, M. I. Katsnelson, I. V. Grigorieva, S. V. Dubonos, and A. A. Firsov, Nature (London) 438, 197 (2005).

${ }^{17}$ Y. Zhang, Y. W. Tan, H. L. Stormer, and P. Kim, Nature (London) 438, 201 (2005).

${ }^{18}$ C. Berger et al., Science 312, 1191 (2006).

${ }^{19}$ K. S. Novoselov, Z. Jiang, Y. Zhang, S. V. Morozov, H. L. Stormer, U. Zeitler, J. C. Maan, G. S. Boebinger, P. Kim, and A. K. Geim, Science 315, 1379 (2007).

${ }^{20}$ A. Geim and K. Novoselov, Nat. Mater. 6, 183 (2007).

${ }^{21}$ Zhihong Chen, Yu-Ming Lin, Michael J. Rooks, and Phaedon Avouris, Physica E (Amsterdam) 40, 228 (2007).

${ }^{22}$ M. Y. Han, B. Ozyilmaz, Y. Zhang, and P. Kim, Phys. Rev. Lett. 98, 206805 (2007).

${ }^{23}$ B. Ozyilmaz, P. Jarillo-Herrero, D. Efetov, D. A. Abanin, L. S. Levitov, and P. Kim, Phys. Rev. Lett. 99, 166804 (2007).

${ }^{24}$ L. A. Ponomarenko, F. Schedin, M. I. Katsnelson, R. Yang, E. H. Hill, K. S. Novoselov, and A. K. Geim, Science 320, 356 (2008).

${ }^{25}$ J. Wu, W. Pisula, and K. Mullen, Chem. Rev. (Washington, D.C.) 107, 718 (2007).

${ }^{26}$ A. L. Vázquez de Parga, F. Calleja, B. Borca, M. C. G. Passeggi, Jr., J. J. Hinarejos, F. Guinea, and R. Miranda, Phys. Rev. Lett. 100, 056807 (2008)

${ }^{27}$ R. Saito, M. S. Dresselhaus, and G. Dresselhaus, Physical Properties of Carbon Nanotubes (Imperial College, London, 1998).

${ }^{28}$ P. K. Wallace, Phys. Rev. 71, 622 (1947).

${ }^{29}$ M. Inui, S. A. Trugman, and E. Abrahams, Phys. Rev. B 49, 3190 (1994).

${ }^{30}$ Gerardo G. Naumis, Phys. Rev. B 76, 153403 (2007).

${ }^{31}$ V. M. Pereira, J. M. B. Lopes dos Santos, and A. H. Castro Neto, Phys. Rev. B 77, 115109 (2008)

${ }^{32}$ H. C. Longuet-Higgins, J. Chem. Phys. 18, 265 (1950).

${ }^{33}$ Elliott H. Lieb, Phys. Rev. Lett. 62, 1201 (1989).
${ }^{34}$ K. Wakabayashi, M. Sigrist, and M. Fujita, J. Phys. Soc. Jpn. 67, 2089 (1998).

${ }^{35}$ Y.-W. Son, M. L. Cohen, and S. G. Louie, Phys. Rev. Lett. 97, 216803 (2006).

${ }^{36}$ Young-Woo Son, M. L. Cohen, and S. G. Louie, Nature (London) 444, 347 (2006).

${ }^{37}$ L. Pisani, J. A. Chan, B. Montanari, and N. M. Harrison, Phys. Rev. B 75, 064418 (2007).

${ }^{38}$ J. Fernández-Rossier, Phys. Rev. B 77, 075430 (2008).

${ }^{39}$ K. Nakada, M. Fujita, G. Dresselhaus, and M. S. Dresselhaus, Phys. Rev. B 54, 17954 (1996).

${ }^{40}$ L. Brey and H. A. Fertig, Phys. Rev. B 73, 235411 (2006).

${ }^{41}$ F. Muñoz-Rojas, D. Jacob, J. Fernández-Rossier, and J. J. Palacios, Phys. Rev. B 74, 195417 (2006).

${ }^{42}$ S. Reich, J. Maultzsch, C. Thomsen, and P. Ordejón, Phys. Rev. B 66, 035412 (2002).

${ }^{43}$ M. P. López Sancho, M. C. Muñoz, and L. Chico, Phys. Rev. B 63, 165419 (2001).

${ }^{44}$ O. Hod, J. E. Peralta, and G. E. Scuseria, Phys. Rev. B 76, 233401 (2007); O. Hod, V. Barone, and G. E. Scuseria, ibid. 77, 035411 (2008).

${ }^{45}$ W. L. Wang, S. Meng, and E. Kaxiras, Nano Lett. 8, 241 (2008); O. V. Yazyev, W. L. Wang, S. Meng, and E. Kaxiras, ibid. 8, 766 (2008).

${ }^{46}$ Vitor M. Pereira, F. Guinea, J. M. B. Lopes dos Santos, N. M. R. Peres, and A. H. Castro Neto, Phys. Rev. Lett. 96, 036801 (2006).

${ }^{47}$ M. Ezawa, Phys. Rev. B 73, 045432 (2006); M. Ezawa, Phys. Status Solidi C 4, 489 (2007).

${ }^{48}$ V. Barone, O. Hod, and G. E. Scuseria, Nano Lett. 6, 2748 (2006).

${ }^{49}$ J. Fernández-Rossier, J. J. Palacios, and L. Brey, Phys. Rev. B 75, 205441 (2007).

${ }^{50}$ F. Muñoz-Rojas, J. Fernández-Rossier, L. Brey, and J. J. Palacios, Phys. Rev. B 77, 045301 (2008).

${ }^{51}$ W. P. Su, J. R. Schrieffer, and A. J. Heeger, Phys. Rev. Lett. 42, 1698 (1979).

${ }^{52}$ L. Pisani, B. Montanari, and N. M. Harrison, New J. Phys. 10, 033002 (2008).

${ }^{53}$ L. Brey, H. A. Fertig, and S. Das Sarma, Phys. Rev. Lett. 99, 116802 (2007).

${ }^{54}$ Saeed Saremi, Phys. Rev. B 76, 184430 (2007).

${ }^{55}$ J. K. Furdyna, J. Appl. Phys. 64, R29 (1988).

${ }^{56}$ H. Boukari, P. Kossacki, M. Bertolini, D. Ferrand, J. Cibert, S. Tatarenko, A. Wasiela, J. A. Gaj, and T. Dietl, Phys. Rev. Lett. 88, 207204 (2002).

${ }^{57}$ J. Fernández-Rossier and L. Brey, Phys. Rev. Lett. 93, 117201 (2004).

${ }^{58}$ S. Sorella and E. Tosatti, Europhys. Lett. 19, 699 (1992).

${ }^{59}$ N. M. R. Peres, M. A. N. Araujo, and D. Bozi, Phys. Rev. B 70, 195122 (2004).

${ }^{60}$ O. V. Yazyev, arXiv:0802.1735 (unpublished). 